\title{
Nanopore Characterization and Optical Modeling of Transparent Polycrystalline Alumina
}

\author{
Michael Stuer,* Paul Bowen, Marco Cantoni, Carlos Pecharroman, and Zhe Zhao*
}

Production of transparent ceramics has become a topic of resurgent interest in recent years, with its promise of near-net shaping appealing to applications ranging from biomedicine to solar energy. However, the mechanisms governing ceramic transparency, translucency, and opaqueness are not entirely understood. Models of both grain boundary and pore scattering have been proposed, but too often without sufficient experimental corroboration. An extensive experimental analysis of transparent alumina samples is presented, establishing a first direct link between the observed transparency, defect size, and porosity. Given the unprecedented experimental detail from the full 3D pore reconstruction from the FIB tomography, how to correctly interpret the experimentally observed transparency is additionally shown. The unprecedented experimental and theoretical agreement for the first time identifies the relative contributions of different scattering mechanisms, thereby paving the way forward for microstructural tuning of transparent polycrystalline alumina.

of sintering transparent ceramics to a near net shape, which has a potentially large economic and design impact, ${ }^{[16]}$ particularly in comparison to machining the single-crystal bulk material. The general trend for reliable, mechanically robust ceramics has driven down grain sizes ${ }^{[17,18]}$ to a regime requiring substantial attention to all the ceramic processing steps, from the powder synthesis to the sintering (i.e., firing). Homogenizing and scaling down the microstructure allows higher densities to be achieved, and thus improves the overall ceramic properties, including optical transparency and, eventually, colored transparent ceramics. ${ }^{[7,19-21]}$

Improving optical transparency proceeds generally by reducing the grain size and the pore fraction in the ceramic, as these both represent scattering sources

\section{Introduction}

Research activity in the fabrication of transparent ceramic materials has seen remarkable growth over the last decades, ${ }^{[1-9]}$ with applications in an impressively broad and ever-expanding range of industries including optoelectronics and lighting, ${ }^{[10]}$ medical and dental implants, ${ }^{[11,12]}$ ceramic armors, ${ }^{[8]}$ and solar energy. ${ }^{[13-15]}$ One key feature and driving force of such activity is the possibility

M. Stuer, Dr. P. Bowen

Powder Technology Laboratory

École Polytechnique Fédérale de Lausanne

1015 Lausanne, Switzerland

E-mail: michael.stuer@a3.epfl.ch

Dr. M. Cantoni

Interdisciplinary Center for Electron Microscopy

École Polytechnique Fédérale de Lausanne

1015 Lausanne, Switzerland

Dr. C. Pecharroman

Instituto de Ciencia de Materiales de Madrid

CSIC, 28049 Madrid, Spain

Dr. Z. Zhao

Department of Materials and Environmental Chemistry

Stockholm University

10691 Stockholm, Sweden

E-mail: zhezhao@kth.se

Dr. Z. Zhao

Department of Materials Science and Engineering

KTH Royal Institute of Technology

Ceramic Technology Division

10044, Stockholm, Sweden

DOI: 10.1002/adfm.201200123 which limit the attainable optical transmittance. For ceramic materials in which the single crystallites are themselves anisotropic, an additional contribution arises from the crystalline birefringence, as the relative orientation between adjacent grains imposes an index mismatch of the ordinary and extraordinary axes at each interface. Each grain boundary therefore represents a scattering source, which can in principle be minimized by introducing texture into the microstructure, ${ }^{[22]}$ such that index mismatch across a grain boundary is minimized and the transmittance thereby improved. This fundamental property of birefringent crystalline structures commonly encountered in standard ceramic materials (e.g., $\mathrm{Al}_{2} \mathrm{O}_{3}$ ) explains the motivation of some researchers to focus on materials with cubic crystalline structures (e.g., $\mathrm{MgAl}_{2} \mathrm{O}_{4}$ ). Despite the general inferiority of the mechanical properties (depending on the grain size) of the isotropic materials, this strategy limits the challenge for improved optical properties to the residual porosity reduction. ${ }^{[8]}$

Specifically for transparent polycrystalline alumina (PCA), the birefringence is small $\left(n_{\mathrm{o}}=1.760, n_{\mathrm{e}}=1.768\right)$ but by no means non-negligible. Experimentally, relatively high real in-line transmittance (RIT) values have been achieved (50-70\% at $640 \mathrm{~nm}$, cf. $86 \%$ for sapphire $)^{[1-4,10]}$ in samples prepared either by natural sintering followed by hot isostatic pressing (post-HIP) ${ }^{[2,6]}$ or by pulsed electric current sintering (PECS), also referred to as spark plasma sintering (SPS). ${ }^{[4]}$ The prevalent optical model to predict the real in-line transmittance (RIT) describes scattering from the grain boundaries quantitatively, but includes the contribution from the residual porosity only qualitatively. This shortcoming has persisted equally on the experimental side, as comprehensive characterization of the pore size distribution 
poses a significant technical challenge. Nonetheless, the importance of its realization is beyond dispute, as without this information and an optical model capable of incorporating it, the multiple scattering sources present in any PCA sample can neither be properly identified nor accordingly addressed in the processing and fabrication steps, limiting understanding and impeding the route to improved transparencies.

Here, an exhaustive analysis of the PCA microstructure by scanning electron microscopy (SEM), transmission electron microscopy (TEM), and 3D focused ion beam (FIB) methods is performed to determine the complete pore characteristics of alumina samples prepared by PECS. The advantage of the PECS approach is that a much better control of grain size at different sintering temperatures or heating rates is possible because of the speed of sintering process $(15 \mathrm{~min})^{[23,24]} \mathrm{com}$ pared to natural sintering and post HIP treatments $(2-4 \mathrm{~h}),{ }^{[2,6]}$ such that samples with nearly identical grain sizes can be obtained for a wide range of heating rates. As such, the distinct degree of heating-rate-dependent coloration observed is correlated only with the porosity level in terms of structural differences among the samples, as we demonstrate by full pore reconstruction by 3D FIB tomography. By measuring the RIT and using the pore and grain size data as experimental inputs, the historically employed optical mode ${ }^{[1,25]}$ can, for the first time, be directly evaluated. Together, these efforts reveal that the model's commonly used characteristic grain and pore size parameters lead to a severe overestimation of the effect of small pores. We demonstrate experimentally that small quantities of ultrafine pores $(<50 \mathrm{~nm})$ have no significant effect on the RIT within the limits of a certain volume concentration. Absorption measurements are also performed in order to provide a complete picture of the factors influencing the transmittance values, which will be discussed in detail in a subsequent publication. Improvements to the scattering model are made by modifying the input parameters based on the analytical pore size distribution characteristics.

\section{Sample Preparation and Experimental Section}

The starting powder (Sumitomo AA04, Solvadis Chemag Gmbh, Frankfurt am Main, Germany) used in these studies was near-spherical, facetted, high-purity $\alpha-\mathrm{Al}_{2} \mathrm{O}_{3}$ with a median particle size, $D_{\mathrm{v} 50}$, of $510 \mathrm{~nm}$, a total impurity concentration of less than 0.01 mass $\%$ ( $\leq 5 \mathrm{ppm}$ for $\mathrm{Si}, \mathrm{Na}, \mathrm{Mg}, \mathrm{Cu}$, and $\mathrm{Fe}$ ), and a specific surface $S_{\mathrm{BET}}$ of $4.2 \mathrm{~m}^{2} \mathrm{~g}^{-1}$. $\mathrm{Mg}^{2+}$ doping at a level of $450 \mathrm{ppm}$ total cationic ratio was introduced through the addition of a Mg-nitrate solution $\left(\mathrm{Mg}\left(\mathrm{NO}_{3}\right) \cdot 6 \mathrm{H}_{2} \mathrm{O}\right.$, Aldrich, Switzerland) to the powder dispersion before freeze drying.

For PECS (Dr. Sinter 2050, Sumitomo Coal Mining Co., Tokyo, Japan) sintering, $0.8 \mathrm{~g}$ of the doped powder was loaded in a graphite dye (inner diameter $12 \mathrm{~mm}$ ), the internal surface of which was covered with a graphite fiber sheet to avoid direct contact between the powder compact and the die. The sintering atmosphere is $10 \mathrm{~Pa}$, and the temperature was measured by an optical pyrometer focused on a small cavity in the graphite die, with a distance between powder and cavity bottom of $5 \mathrm{~mm}$. For maximum reproducibility, the sintering temperature and pressure were controlled by automatic controller units, with a
Table 1. Summary of the sintering parameters for the sample series investigated.

\begin{tabular}{lcccc}
\hline Sample type & A & B & C & D \\
\hline Soak temperature $\left[{ }^{\circ} \mathrm{C}\right]$ & \multicolumn{4}{c}{1300} \\
Soak pressure $[\mathrm{MPa}]$ & \multicolumn{4}{c}{100} \\
Heating rate $\left[{ }^{\circ} \mathrm{C} \mathrm{min}^{-1}\right]$ & 100 & 233 & 350 & 100 \\
Pressure application & During the whole sintering cycle & At soak temperature \\
\hline
\end{tabular}

pulse sequence for all the samples of 12:2 (i.e., 12 on/2 off). With this method, a series of samples was prepared using three different heating rates $\left(100,233\right.$, and $\left.350^{\circ} \mathrm{C} \mathrm{min}-1\right)$ up to the dwell temperature of $1300{ }^{\circ} \mathrm{C}$ and two different moments of the $100 \mathrm{MPa}$ pressure application (during the temperature ramp or only upon reaching the soak temperature), as summarized in Table 1 . The average grain size across all the resulting samples was $680 \mathrm{~nm} \pm 30 \mathrm{~nm}$.

After sintering, the samples were polished down to a thickness of $1 \mathrm{~mm}$ to obtain mirror-like parallel surfaces. Transmittance measurements were performed with a spectrometer (UV-VIS-NIR Lambda 900, PerkinElmer, USA) in the wavelength range from 300 to $1000 \mathrm{~nm}$. To measure only the RIT light, two metallic shields each with a hole of $4 \mathrm{~mm}$ diameter were inserted in the light path. The RIT values for all the samples were in the range of $51 \% \pm 4 \%$ after sintering, with the lower RIT numbers corresponding to the higher heating rates.

Thermal treatment (TT) of the polished sample series A-D was then performed in air at a heating rate of $10{ }^{\circ} \mathrm{C} \mathrm{min}^{-1}$ to $1150^{\circ} \mathrm{C}$ for $30 \mathrm{~min}$ before natural cooling. Because the temperature of this thermal etch is insufficient to promote grain growth in PCA, the grain size remains unchanged at approximately $680 \mathrm{~nm}$, but a significant degree of coloration appears across the sample series. As pictured in Figure 1, samples A-C, where the pressure was applied during the entire sintering cycle, display a distinct yellow tone, which is more severe for the faster heating rates, in correlation with the lower pre-thermal treatment RIT values. On the other hand, sample D, sintered with the pressure applied only at the dwell temperature, still appears colorless after the thermal treatment.

For quantitative investigation of the effect of porosity on the optical transmittance, a series of "C-type" samples was prepared by PECS: one as-sintered (C1), a second with a $1150{ }^{\circ} \mathrm{C}$ thermal treatment resulting in strong coloration $(\mathrm{C} 2)$, and a third with this same thermal treatment followed by a hot isostatic press (post-HIP) treatment (C3). The post-HIP treatment, carried out at $1200{ }^{\circ} \mathrm{C}$ and 1500 bar, largely reverses the porosity generated by the thermal treatment. It should be noted that each sample of this C-series was performed after its final processing step, so that there is no contribution from a difference in surface roughness between the three. These samples were subsequently characterized by RIT, SEM, TEM, and 3D FIB tomography. SEM pictures (JSM-7000F, JEOL, Japan) were taken on cross-section polished samples (SM-09010, JEOL, Japan) and TEM pictures (JEM-2100, JEOL, Japan) obtained on samples prepared by ion slicing (EM-09100IS, JEOL, Japan). FIB Nano-Tomography (NVision 40 focused ion beam system, Zeiss, Germany) 

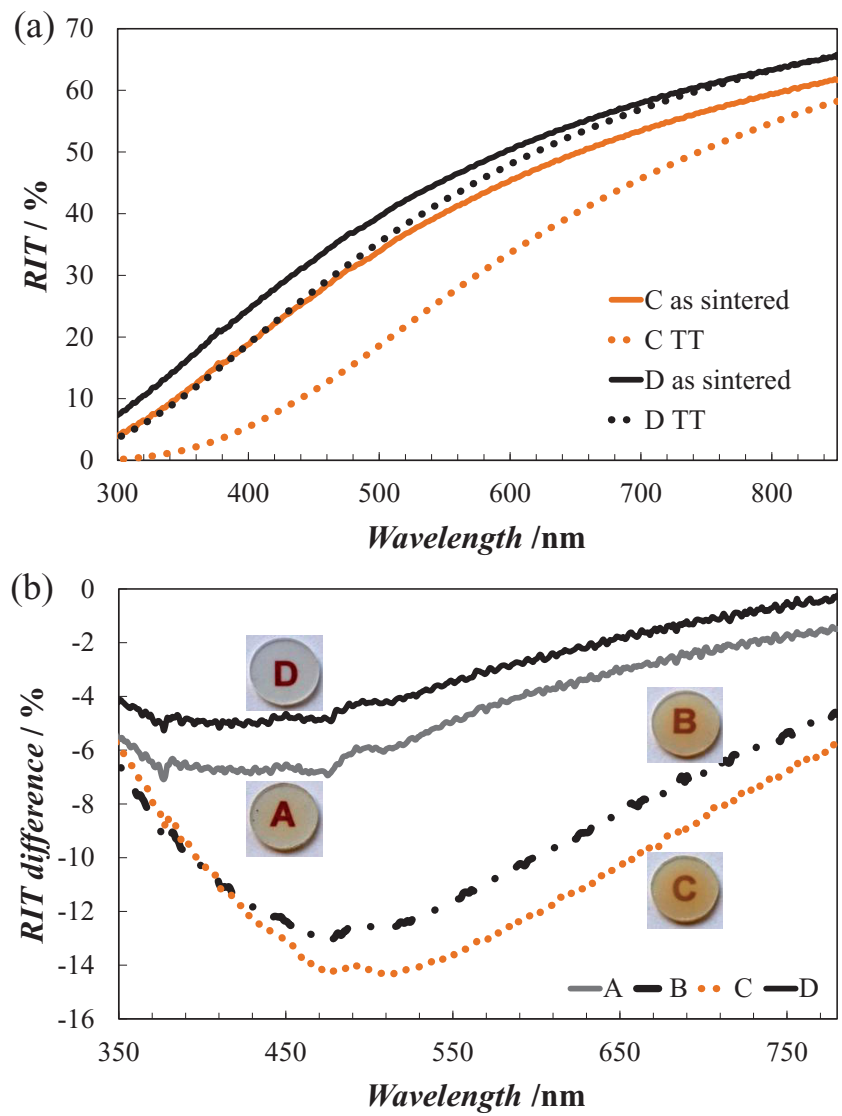

Figure 1. a) Raw RIT spectral data shown only for samples $C$ and $D$ for clarity, both as-sintered and after thermal treatment (TT) at $1150{ }^{\circ} \mathrm{C}$ for $30 \mathrm{~min}$. b) RIT change over the visible range for all of the samples A-D after the $\Pi T$ with respect to the as-sintered samples. The sintering parameters summarized in Table 1. The images show the increasingly severe degree of yellow sample coloration after the TT for samples A-C accompanying the inhomogeneous RIT loss over the visible spectrum.

was performed on gold-coated bulk samples by Ga-ion milling with a voxel size of $10 \mathrm{~nm} \times 10 \mathrm{~nm} \times 10 \mathrm{~nm}$. The total acquired sample volume varied depending on the sample between $400 \mu \mathrm{m}^{3}$ and $800 \mu \mathrm{m}^{3}$. The resulting image stack was treated and analyzed using the Fiji package of ImageJ.

\section{Experimental Results}

Based on the historical model of the RIT, ${ }^{[1,25]}$ the pre-thermal treatment transmittance values of $51 \% \pm 4 \%$ across samples A-D imply that they should also have residual porosity levels within $<0.001 \%$ of each other and a median pore volume diameter $\left(D_{\mathrm{v} 50}\right)$ of less than $50 \mathrm{~nm}$. The yellowish coloration that appears after the 30 min thermal etching step at $1150{ }^{\circ} \mathrm{C}$ of course dramatically degrades the RIT values of these thermally treated samples, a change which is quantified by determining the difference in the RIT between the pre- and post-etched samples over the visible range of wavelengths (Figure 1).

From these measurements, the yellow coloration of the samples seen in Figure 1 is accompanied by an inhomogeneous RIT loss over the visible spectrum, with the strongest coloration of sample $\mathrm{C}$ corresponding to a maximum loss in the blue near $490 \mathrm{~nm}$. TEM investigations before thermal treatment revealed many intra-granular dislocation walls (Figure 2) in the C-type samples, i.e., those with the highest heating rates $\left(350{ }^{\circ} \mathrm{C} \mathrm{min}^{-1}\right)$, which in the course of the thermal treatment developed the strongest coloration. Such pre-treatment defects could not be observed for sample $\mathrm{D}$, which indeed developed no coloration at all.

Because the thermal etching, which permits the observation of the grain boundaries, works to obscure the visibility of the pores created by the etching itself, no pores could be observed on the surface of these thermally etched samples. Therefore instead of the traditional mechanical polishing followed by thermal etching, cross-section ion polishing of the samples after thermal treatment was used to reveal the small pores in the SEM for sample C (Figure 3a) and sample D (Figure 3b). The high channeling contrast ${ }^{[26]}$ resulting from a better preservation of the underlying crystal structure during ion polishing revealed pores situated at the triple boundaries, approximately $50 \mathrm{~nm}$ in diameter. In this way, a distinct difference in the porosity of the thermally treated samples could be observed between samples $\mathrm{C}$ and $\mathrm{D}$, directly linking the pre-treatment dislocations of sample $\mathrm{C}$ to the appearance of pores. In contrast, almost no pores were observed in the colorless sample D.

Observation of varying pore content from the 2D surface, however, does not properly characterize the total pore content and its size distribution, the critical input for modeling the RIT. Extracting a pore size from the SEM images and determining the total pore content by the usual Archimedes method is not possible, especially because of the insufficient sensitivity of conventional high precision balances and the uncertainties of using accurate theoretical density values for fine-grained ceramic samples. The finer the ceramic microstructure, the larger are the grain boundary surfaces within the sample, representing local crystallographic defects affecting the theoretical density. Serial sectioning by FIB tomography was therefore employed to

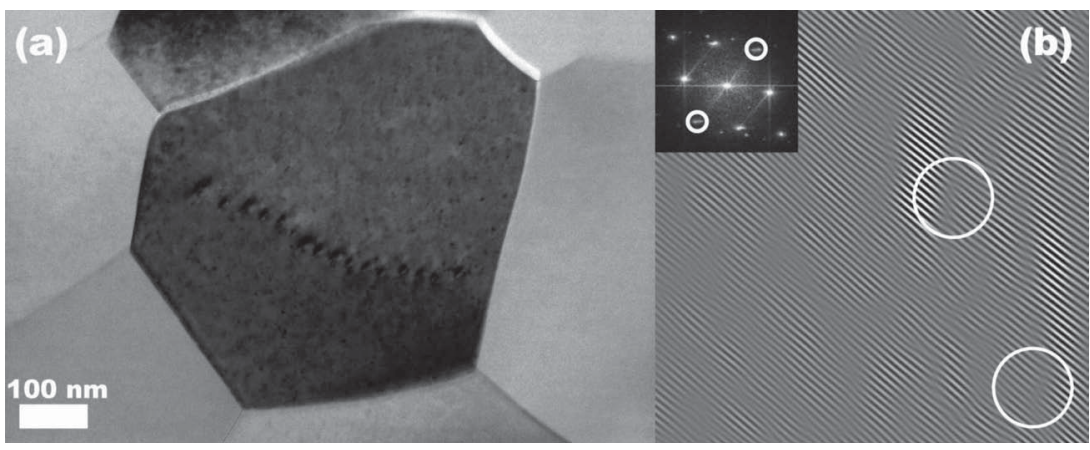

Figure 2. a) TEM image of sample $C$ (cf. Figure 1) before thermal treatment. The image shows a dislocation wall within the grain, hypothesized to form pores during the subsequent thermal treatment. b) Fast Fourier transform analysis of a high-resolution TEM image showing the presence of defects (dislocations) from irregularities in the periodicity. 

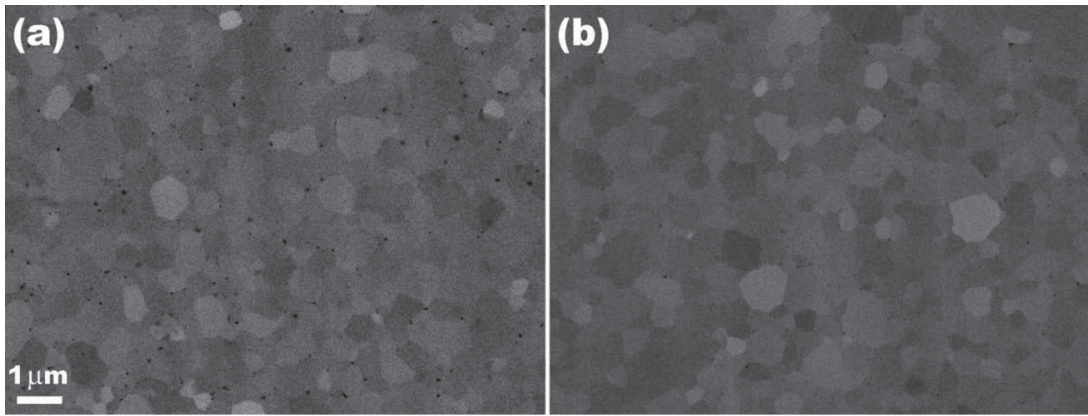

Figure 3. SEM images of a) C-type and b) D-type samples thermally treated before cross section ion polishing. The impressive channeling contrast reveals a clear positioning of the pores at the triple joints in the microstructure.

obtain a full $3 \mathrm{D}$ reconstruction of the pores, thus permitting a complete analysis of the pore fraction, size, and distribution.

To this end, a new series of C-type (see Figure 1) samples was prepared specifically to obtain a range of porosity content to measure by serial FIB sectioning. We refer to the samples as: $\mathrm{C} 1$, as sintered; $\mathrm{C} 2$, sintered and thermally etched; and C3, sintered, thermally etched and post-HIPed. Representations of the 3D FIB reconstructions are shown for a $4.11 \times 4.11 \times 4.11 \mu \mathrm{m}^{3}$ volume in Figure 4 and the corresponding distribution data are

\section{Discussion}

Interestingly, sample C1 (the as-sintered state), with a total porosity of 0.036 vol\%, follows the historically used optical model for the RIT of anisotropic ceramics described by Apetz and van Bruggen for 0 vol\% porosity ${ }^{[1]}$ (Figure 6). However, taking into account the pore scattering, as these authors discussed qualitatively ${ }^{[1]}$ based on earlier suggestions by Peelen and Metselaar, ${ }^{[25]}$ the observed porosity determined from the $3 \mathrm{D}$ FIB tomography should reduce the RIT to a mere $\approx 10 \%$.

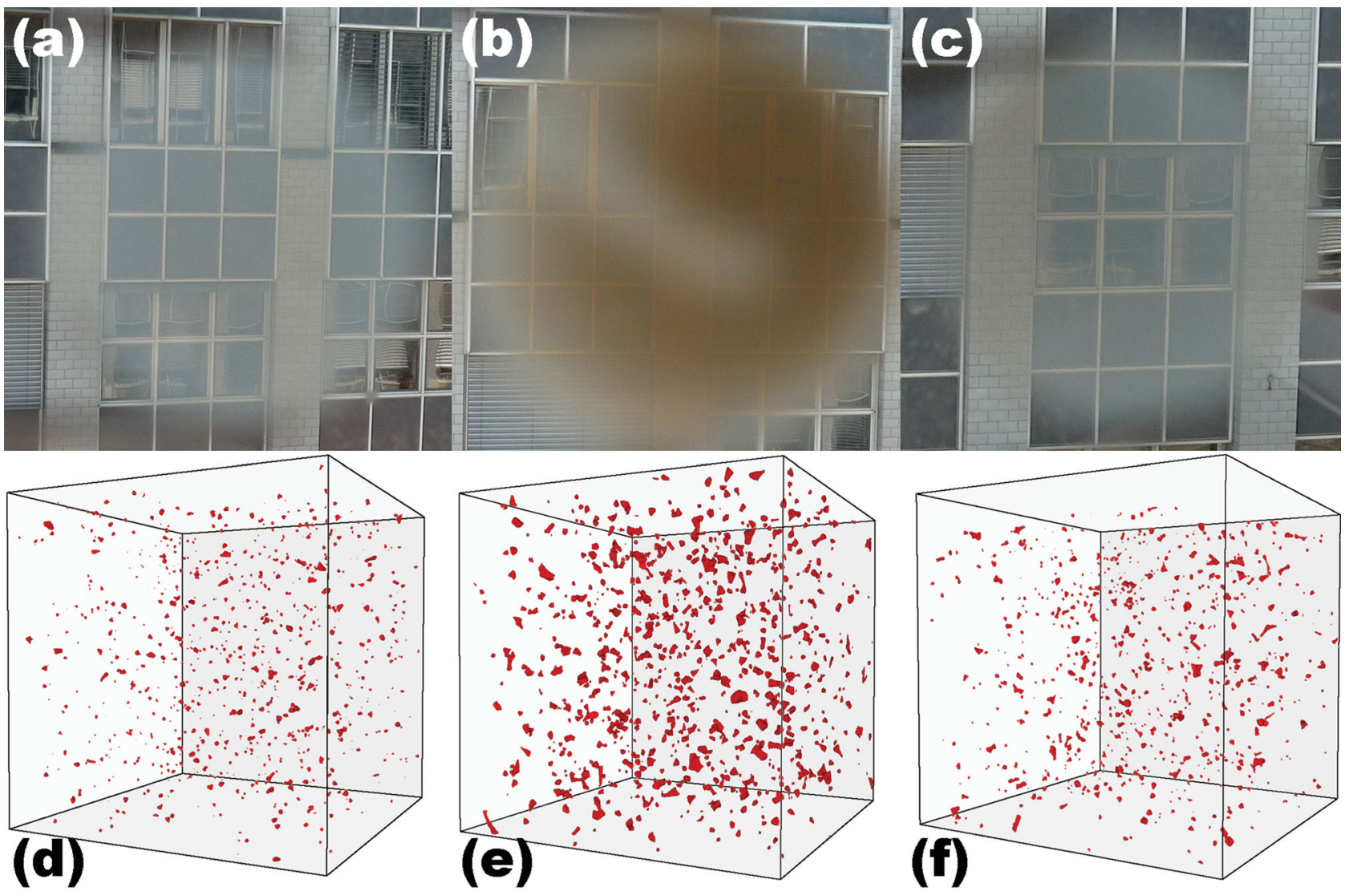

Figure 4. Sample images $(a-c)$ and 3D-FIB tomography representations $(d-f)$ of the porosity of the different treatment states: a,d) as-sintered (sample $\mathrm{Cl}$ ); b,e) after thermal treatment at $1150^{\circ} \mathrm{C}$ (sample C2); and c,f) after thermal treatment followed by post-HIP at 1500 bar (sample C3). The 3D-FIB pore reconstruction is of a $4.11 \times 4.11 \times 4.11 \mu \mathrm{m}^{3}$ volume for all the samples. Note that the uncolored area in the middle of sample C2 (b) resulted from dislocation release into a crack present in the sample before thermal treatment. 


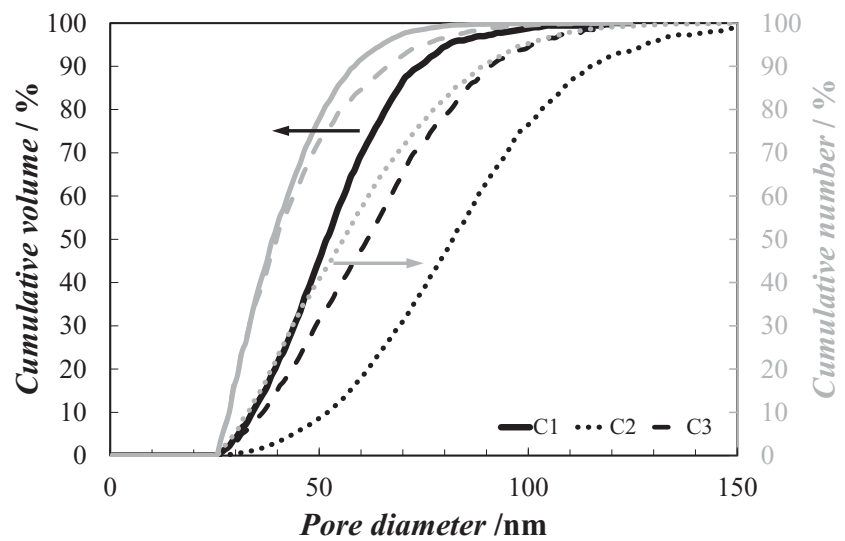

Figure 5. Volume (black lines) and number (gray lines) pore size distributions for samples $\mathrm{Cl}, \mathrm{C} 2$, and $\mathrm{C} 3$ obtained from the 3D FIB tomography. Note the distribution shift towards smaller characteristic pore diameters when switching from the volume to the number distribution.

The measured RIT value of $49 \%$ is thus much higher than predicted, clearly demanding an optical model refinement.

The RIT, i.e., the fraction of light passing through the sample without being absorbed or scattered, is generally described by the Lambert-Beer law:

$\mathrm{RIT}=\left(1-R_{\mathrm{S}}\right) \exp \left(-\gamma_{\mathrm{tot}} d\right)$

where $R_{\mathrm{S}}$ describes the reflection losses from both sample surfaces (i.e., 0.14 for alumina), $d$ is the sample thickness, and $\gamma_{\text {tot }}$ is the total loss coefficient. This $\gamma_{\text {tot }}$ parameter includes the scattering from grain boundaries as well as from pores, and can be represented as a summation of these effects:

$\gamma_{\mathrm{tot}}=\gamma_{\mathrm{gb}}+\gamma_{\mathrm{pore}}+\ldots$

with $\gamma_{\mathrm{gb}}$ the scattering coefficient from the grains and $\gamma_{\text {pore }}$ the scattering coefficient from the pores. It should be noted that any number of supplementary loss contributions may be present, including a pure absorption term from optical centers resulting

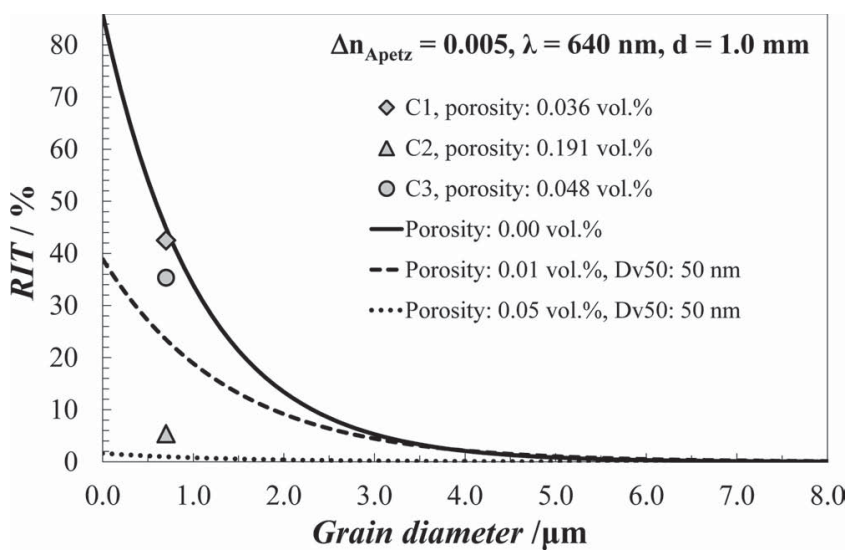

Figure 6. RIT model predictions of Apetz and van Bruggen ${ }^{[1]}$ as a function of the grain diameter and porosity levels up to 0.05 vol\% for the $1.0 \mathrm{~mm}$ thick samples at $640 \mathrm{~nm}$. The experimental RITs for $\mathrm{C} 1, \mathrm{C} 2$, and $\mathrm{C} 3 \mathrm{sam}$ ples are represented to compare the predicted and the measured RITs. from oxygen vacancies or dopant cations. Historically, however, these have also been categorically neglected.

Although several authors ${ }^{[1,7,9,25]}$ suggest how to approximately address porosity effects in their models, they show the validity of their $\gamma_{\mathrm{gb}}$ expressions on a set of samples assumed to be fully dense by standard density measurement techniques, due to the difficulties of characterizing residual porosity, and therefore neglect the $\gamma_{\text {pore }}$ contribution from pores, only qualitatively discussing its effects. In fact, Peelen and Metselaar calculated a scattering cross section for pores based on Rayleigh scattering, ${ }^{[27]}$ neglecting the contribution of the grain boundary scattering. For pores strictly smaller than the incident wavelength, they indicate:

$\gamma_{\text {pore, Peelen }}=\frac{3 V_{\mathrm{p}} Q_{\mathrm{eff}}}{4 r_{\mathrm{m}} \exp \left(3.5 \sigma^{2}\right)}$

where $V_{\mathrm{p}}$ is the specific pore volume, $r_{\mathrm{m}}$ is the radius of the mode of the volume pore size distribution, $\sigma$ is the standard deviation factor for a log normal distribution of pores, and $Q_{\text {eff }}$ is an effective dimensionless scattering efficiency of the pores.

Today, the most widely employed expression of the scattering coefficient from the grains $\left(\gamma_{\mathrm{gb}}\right)$ was proposed nearly a decade ago by Apetz and van Bruggen. ${ }^{[1]}$ Based on Rayleigh-GansDebye ${ }^{[27,28]}$ scattering, they proposed that:

$\gamma_{\mathrm{gb}, \mathrm{Apetz}}=3 \frac{\pi^{2} r}{\lambda_{0}^{2}} \Delta n^{2}$

where $r$ is the (average) grain radius, $\Delta n$ is the (average) refractive index change between two adjacent grains $\left(\Delta n_{\max }=n_{\mathrm{o}}-\right.$ $\left.n_{\mathrm{e}}=0.008\right)$, and $\lambda_{0}$ is the wavelength of the incident light in vacuum. From this expression, valid for grain sizes of the order of magnitude of the incident wavelength, the influence of the grain size, and the degree of crystalline birefringence are both immediately evident.

With the enumerated characteristic pore diameter, i.e., the mode of the volume pore size distribution, the model predicts an extremely high sensitivity of the RIT on the residual porosity. Porosities as low as 0.05 vol\% are predicted to be sufficient to completely nullify the RIT (Figure 6). Because of the lack of accurate porosity characterization to date, the validity of this pore scattering coefficient has not been investigated before the present work. This is mainly due to the technical difficulties of the quantitative characterization of such low porosity contents. This is especially true given that not only the total volume pore content must be measured, but also the respective sizes and distributions.

Given this discrepancy between model predictions and the experimental results in the light of our 3D FIB tomography pore analysis, a more recently introduced grain boundary scattering coefficient ${ }^{[22]}$ and a modified pore scattering coefficient were employed to describe the optical transmission of these transparent PCA samples. The grain scattering $\left(\gamma_{\mathrm{gb}}\right)$ and pore scattering $\left(\gamma_{\text {pore }}\right)$ coefficients, in this new approach, are based on the same Rayleigh and Rayleigh-Gans scattering approximations, respectively:

$\gamma_{\mathrm{gb}, \text { Pecharroman }}=6 \frac{\pi^{2} \alpha(\xi)\left\langle a_{\mathrm{g}}\right\rangle}{\lambda_{0}^{2}} \Delta n^{2}$ 


$$
\gamma_{\mathrm{p}, \text { Pecharroman }}=32 \frac{f \pi^{4}\left\langle a_{\mathrm{p}}^{3}\right\rangle}{\lambda_{0}^{4}}\left(\frac{n^{2}-1}{n^{2}+2}\right)^{2}
$$

with $\alpha(\xi)$ a texture function resulting from the Rayleigh-GansDebye approximation dependent on the preferential textural angle $\xi ; f$ the pore volume fraction, $n$ the average refractive index of the ceramic matrix, and $\left\langle a_{\mathrm{g}}\right\rangle$ and $\left\langle a_{\mathrm{p}}\right\rangle$ the characteristic grain and pore radii, respectively, defined by:

$$
\begin{aligned}
& \left\langle a_{\mathrm{g}}\right\rangle=\sum_{i} a_{\mathrm{g}, i} f_{i}=\frac{\sum_{i} a_{\mathrm{g}, i}^{4}}{\sum_{i} a_{\mathrm{g}, i}^{3}} \\
& \left\langle a_{\mathrm{p}}^{3}\right\rangle=\frac{\sum_{i} a_{\mathrm{p}, i}^{6}}{\sum_{i} a_{\mathrm{p}, i}^{3}}
\end{aligned}
$$

where $a_{\mathrm{g}, \mathrm{i}}$ and $a_{\mathrm{p}, \mathrm{i}}$ are the grain and pore radius, respectively, of the $i^{\text {th }}$ class from the size distribution, $f_{i}$ is the fraction of grains with radius $a_{\mathrm{g}, \mathrm{i}}$, and $\Sigma_{i}$ is the sums over the whole population of the grain and pore size distributions. These characteristic radii are calculated directly from the full distribution of grain and pore sizes. Note that the pore scattering coefficient is highly sensitive to the pore size because of its cubic dependence.

In these new expressions, the grain scattering coefficient is similar to the one earlier proposed by Apetz and van Bruggen, except for the texture function that has been separated from the $\Delta n$. The essential difference between the former and these new expressions is the definition of the characteristic grain and pore sizes. As seen from Figure 5, the $\left\langle a_{\mathrm{g}}\right\rangle$ and $\left\langle a_{\mathrm{p}}\right\rangle$ sizes generally fall within the range of $D_{\mathrm{n} 90}$ to $D_{\mathrm{n} 99}$ values of the numerical grain size and pore size distributions from 3D FIB results, with the exact value depending on the span of the distribution of grain and pore sizes. The $D_{\mathrm{n} 90}$ value describes the diameter of the grain or pore at which $90 \%$ have a size lower than $D_{\mathrm{n} 90}$. Since the number distribution is always smaller than the volume distribution, taking the average grain size (Apetz) ${ }^{[1]}$ or the maximum grain size observed (Pecharroman) ${ }^{[22]}$ gives similar values if no abnormal grain growth is observed. Note that the use of the number distribution can be logically derived because of the integer character of the scattering events.

With these modified expressions, the agreement between the measured RITs and the ones predicted from the model is dramatically improved, as shown in Figure 7. Here all the measured porosity levels are used as inputs to the Pecharroman model, resulting in excellent agreement with the observed 42.5\% RIT for the as-sintered C1 sample with its 0.036 vol\% porosity and $\left\langle a_{\mathrm{p}}\right\rangle=29 \mathrm{~nm}$. This is in comparison to the approximately 10\% RIT that would be predicted from the Apetz model with this residual porosity level. The Pecharroman inputs result in a slight overestimation of the observed $35.3 \%$ RIT value for the post-HIPed sample C3, and a more severe overestimation of the observed 5.4\% RIT for the thermally treated sample C2 with its high porosity. However, for C2 the predictions have been greatly improved compared to the historical pore scattering expression, which predicts an RIT of $0 \%$. The discrepancy still observed for $\mathrm{C} 2$ between the model prediction and the experimental data is at least partially due to limits of the Rayleigh-Gans approximation used for the grain-boundary

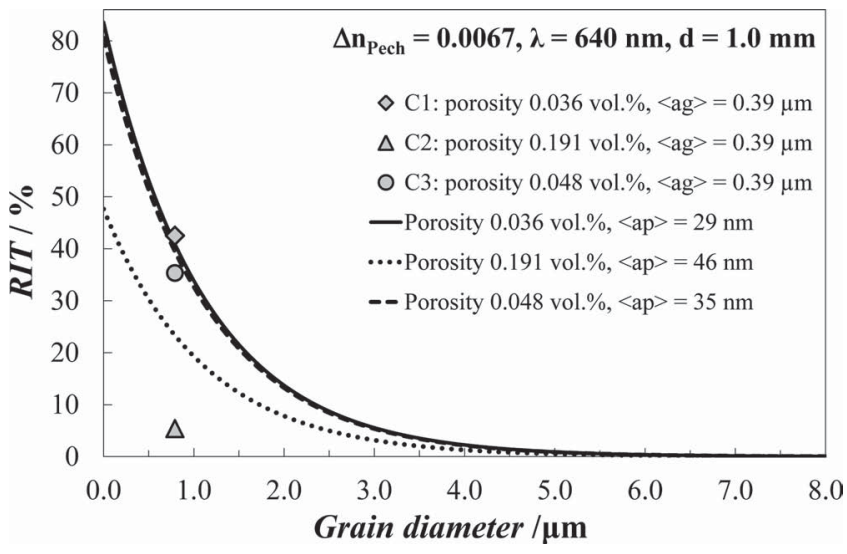

Figure 7. RIT as a function of the grain diameter calculated from the model described by Pecharroman et al.[22] The characteristic pore radii used for the calculations $\left\langle a_{\mathrm{p}}\right\rangle$ are taken from the 3D-FIB tomography. The actual measured porosity levels are used for the model input, resulting in excellent agreement for sample $\mathrm{Cl}$ and a slight overestimation of the RIT for sample C3. For C2, the RIT predictions are largely improved compared to the null expectation of the Apetz model.

scattering coefficient calculation because of the extreme 0.191 vol\% porosity content. This approximation effectively puts an upper limit on the pore size that can be accommodated by the model for a given porosity level (see Supporting Information S1), above which accurate modeling must revert to the full Mie scattering solutions.

For sample C3 and partially for sample $\mathrm{C} 2$, the remaining discrepancy between the Pecharroman model predictions and the experimental observations may be attributed to absorption losses (see Supporting Information S2). The results of the absorption measurements together with the discussion of its implications for the sample coloration are beyond the scope of this paper and will be discussed in a separate publication. The presence of an absorption term, however, immediately illustrates that a complete discussion of the optical performance of PCA must also account for active optical centers. Further studies will be crucial to elucidate the relative effects on the observed coloration.

\section{Conclusions}

Comprehensive characterization of the porosity by 3D FIB tomography has allowed the validation of new characteristic pore and grain diameters for optical modeling of PCA and birefringent materials in general. The present work has clearly demonstrated that due to the use of inaccurate characteristic sizes, the commonly employed RIT model ${ }^{[1]}$ slightly underestimates the effect of the grain size but severely overestimates the effect of porosity on the RIT. Despite the generally admitted contrary assumption, porosity hardly affects the RIT below a critical pore size of about $50 \mathrm{~nm}$, depending on the residual pore content, a result that may well alter the trajectory of transparent polycrystalline materials research.

Further improvements to the transparency rely on the reduction of the final grain sizes and/or texturization of the 
microstructure to reduce the effect of the birefringence. The grain size reduction will require the development of novel high purity alumina powders with particle diameters below $100 \mathrm{~nm}$, while texturization may be introduced by colloidal processing and grain alignment in high magnetic fields. ${ }^{[29]}$ One intriguing question that arises particularly in light of the direct observation of an absorption contribution to the RIT is whether a low level of porosity could itself introduce sample coloration due to inhomogeneous RIT losses over the visible spectrum. In any case, with the deeper insights into light scattering in polycrystalline alumina from the FIB tomography presented here, the path is clear on how to improve transmittance for PCA, with the challenge remaining to do so.

\section{Supporting Information}

Supporting Information is available from the Wiley Online Library or from the author.

\section{Acknowledgements}

The Swiss National Science Foundation is acknowledged for grant $n^{\circ}$ 200021-122288/1. The authors thank Cheuk-Wei Tei and Cécile Hébert for TEM and general electron microscope support. The authors acknowledge Gustavo Mata Osoro for additional RIT measurements and Dr. Georges Wagnières and Stefan Geissbühler for equipment and assistance with the absorption characterization.

Received: January 14, 2012 Published online:

[1] R. Apetz, M. P. B. van Bruggen, J. Am. Ceram. Soc. 2003, 86, 480.

[2] A. Krell, P. Blank, H. W. Ma, T. Hutzler, M. P. B. van Bruggen, R. Apetz, J. Am. Ceram. Soc. 2003, 86, 12.

[3] A. Krell, J. Klimke, J. Am. Ceram. Soc. 2006, 89, 1985.

[4] M. Stuer, Z. Zhao, U. Aschauer, P. Bowen, J. Eur. Ceram. Soc. 2010, 30, 1335 .
[5] S. Grasso, B. N. Kim, C. F. Hu, G. Maizza, Y. Sakka, J. Am. Ceram. Soc. 2010, 93, 2460.

[6] A. Krell, P. Blank, H. W. Ma, T. Hutzler, M. Nebelung, J. Am. Ceram. Soc. 2003, 86, 546.

[7] A. Krell, T. Hutzler, J. Klimke, J. Eur. Ceram. Soc. 2009, 29, 207.

[8] A. Krell, J. Klimke, T. Hutzler, J. Eur. Ceram. Soc. 2009, 29, 275.

[9] A. Krell, J. Klimke, T. Hutzler, Opt. Mater. 2009, 31, 1144.

[10] G. C. Wei, J. Phys. D Appl. Phys. 2005, 38, 3057.

[11] M. Andersson, A. Oden, Acta Odontol. Scand. 1993, 51, 59.

[12] L. Sedel, Clin. Orthop. Relat. Res. 2000, 379, 48.

[13] G. C. Bazan, J. Peet, J. Y. Kim, N. E. Coates, W. L. Ma, D. Moses, A. J. Heeger, Nat. Mater. 2007, 6, 497.

[14] Y. S. Wang, D. Q. Chen, Y. L. Yu, P. Huang, F. Y. Weng, Opt. Lett. 2008, 33, 1884

[15] M. Liu, Y. L. Lu, Z. B. Xie, G. M. Chow, Sol. Energy Mater. Sol. Cells 2011, 95, 800.

[16] A. Ikesue, Y. L. Aung, J. Am. Ceram. Soc. 2006, 89, 1936.

[17] J. Seidel, N. Claussen, J. Rodel, J. Eur. Ceram. Soc. 1995, 15, 395.

[18] J. Seidel, N. Claussen, J. Rodel, J. Eur. Ceram. Soc. 1997, 17, 727.

[19] F. D. P. Pires-De-Souza, L. A. Casemiro, L. D. R. Garcia, D. R. Cruvinel, J. Prosthet. Dent. 2009, 101, 13.

[20] Z. Zhao, C. Wang, Mater. Res. Bull. 2010, 45, 1127.

[21] R. Hickel, J. Eur. Ceram. Soc. 2009, 29, 1283.

[22] C. Pecharroman, G. Mata-Osoro, L. A. Diaz, R. Torrecillas, J. S. Moya, Opt. Express 2009, 17, 6899.

[23] Z. J. Shen, Z. Zhao, H. Peng, M. Nygren, Nature 2002, 417, 266.

[24] V. Buscaglia, M. T. Buscaglia, M. Viviani, L. Mitoseriu, A. Testino, P. Nanni, Z. Zhao, M. Nygren, C. Harnagea, D. Piazza, C. Galassi, Phys. Rev. B 2006, 73.

[25] J. G. J. Peelen, R. Metselaar, J. Appl. Phys. 1974, 45, 216.

[26] G. E. Lloyd, Mineral. Mag. 1987, 51, 3.

[27] C. F. Bohren, D. R. Huffman, Absorption and Scattering of Light by Small Particles, Wiley, Chichester, UK 1998.

[28] Light Scattering by Small Particles, (Ed: H. C. van de Hulst), Dover Publications, New York 1981.

[29] N. Terada, H. S. Suzuki, T. S. Suzuki, H. Kitazawa, Y. Sakka, K. Kaneko, N. Metoki, J. Phys. D: Appl. Phys. 2009, 42, 105404. 